\title{
Correction to: Web Information Systems and Technologies
}

\author{
Alessandro Bozzon, Francisco José Domínguez Mayo \\ and Joaquim Filipe
}

\section{Correction to:}

\section{A. Bozzon et al. (Eds.): Web Information Systems and Technologies, LNBIP 399, https://doi.org/10.1007/978-3-030-61750-9}

In the originally published version of the book there was an error in the first name of the second volume editor: "José Francisco Domínguez Mayo" should have been "Francisco José Domínguez Mayo". This has now been corrected. 